\title{
Fact Sheet of Rhino in Nepal
}

- Madhav Khadka*, Bishnu Prasad Thapaliya*

Nepali Name - Gaida

English Name - Greater one horned Rhinoceros

Scientific name - Rhinoceros unicornis

\section{Distribution}

Globally

- India and Nepal

Nepal

- Chitwan National Park (CNP),Bardia

National park (BNP), Suklaphanta Wildlife Reserve (SWR)

Preferred Habitat-Floodplain, grassland and swampy area of Tarai

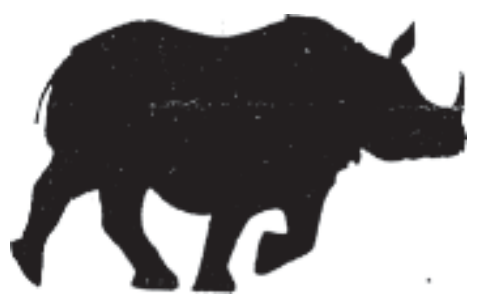

Rhino Census in Nepal

\section{Biology,size and sexual Dimorphism}

Fourth largest living terrestrial mammal

Height - 1 to 2 meter

Length - 4 meter

Weight - 2000 to $3000 \mathrm{~kg}$

Body Color - Light Black

Body Texture - Armour-plated appeared with large tubercles on bare skin

Breeding Age - after 6 years

Gestation Period - 16 month

Inter-birth Interval - 1 Calf every 3 year

(Calves separate from their mother after four years)

Life span - 40 to 70 years

Rhinoceros horn is composed entirely of

densely appressed hairs

Rhinoceros horn size 20 to $60 \mathrm{~cm}$

\begin{tabular}{|cll|}
\hline Year & Protected Area & No. of Rhino \\
\hline 1994 & Chitwan National Park & $446-466$ \\
2000 & Chitwan National Park & 544 \\
& Bardia National Park & 63 \\
& Suklaphanta Wildlife Reserve & 5 \\
2005 & Chitwan National Park & 372 \\
& Bardia National Park & 31 \\
& Suklaphanta Wildlife Reserve & 7 \\
\hline
\end{tabular}

Translocation of Rhino from CNP

\begin{tabular}{|lllll|}
\hline Year & Male & Female & Destination & Total \\
\hline 1986 & 8 & 5 & BNP & 13 \\
1991 & 8 & 17 & BNP & 25 \\
1999 & 4 & - & BNP & 4 \\
$03 / 2000$ & 5 & 5 & BNP & 10 \\
$11 / 2000$ & 3 & 3 & BNP & 6 \\
$11 / 2000$ & 1 & 3 & SWR & 4 \\
2001 & 2 & 3 & BNP & 5 \\
2002 & 5 & 5 & BNP & 10 \\
2003 & 3 & 7 & BNP & 10 \\
Total & 39 & $\mathbf{4 8}$ & & $\mathbf{8 7}$ \\
\hline
\end{tabular}

Rhino Mortality

\begin{tabular}{|l|l|l|l|l|l|l|l|}
\hline Fiscal Year & \multicolumn{3}{|l|}{ Natural Death } & \multicolumn{2}{l|}{ Poaching } & Total \\
\hline & CNP & BNP & SWR & CNP & BNP & SWR & \\
\hline $2056 / 057$ & 28 & - & - & - & 17 & - & 45 \\
$2057 / 058$ & 13 & 2 & - & 12 & 3 & - & 30 \\
$2058 / 059$ & 12 & 2 & - & 38 & 3 & - & 55 \\
$2059 / 060$ & 22 & - & - & 20 & 8 & - & 50 \\
$2060 / 061$ & 19 & - & - & 5 & 5 & - & 29 \\
$2061 / 062$ & 11 & - & 1 & 16 & - & - & 28 \\
$2062 / 063$ & 9 & - & - & 9 & - & - & 18 \\
$2063 / 064$ & 2 & 6 & 2 & 14 & - & - & 24 \\
\hline Total & $\mathbf{1 1 6}$ & $\mathbf{1 0}$ & $\mathbf{3}$ & $\mathbf{1 1 4}$ & $\mathbf{3 6}$ & - & $\mathbf{2 7 9}$ \\
\hline
\end{tabular}

${ }^{*}$ Kathmandu Forestry College, Kathmandu, Nepal 